\title{
Collaborative spinal robot system for laminectomy: a preliminary study
}

\author{
*Zhuofu Li, MD, ${ }^{1-3}$ Shuai Jiang, MD,,-3 Xiongkang Song, MS, ${ }^{4}$ Shanshan Liu, MD,,-3 \\ Chengxia Wang, MD, ${ }^{1-3}$ Lei Hu, PhD, ${ }^{4}$ and Weishi Li, MD ${ }^{1-3}$ \\ 1Department of Orthopaedics, Peking University Third Hospital, Beijing; '2Beijing Key Laboratory of Spinal Disease Research, \\ Beijing; ${ }^{3}$ Engineering Research Center of Bone and Joint Precision Medicine, Ministry of Education, Beijing; and ${ }^{4}$ Robotics \\ Institute, Beihang University, Beijing, China
}

\begin{abstract}
OBJECTIVE The application of robots in the field of pedicle screw placement has achieved great success. However, decompressive laminectomy, a step that is just as critical as pedicle screw placement, does not have a mature robot-assisted system. To address this lack, the authors designed a collaborative spine robot system to assist with laminectomy. In this study, they aimed to investigate the reliability of this novel collaborative spinal robot system and compare it with manual laminectomy (ML).
\end{abstract}

\begin{abstract}
METHODS Thirty in vitro porcine lumbar vertebral specimens were obtained as experimental bone specimens. Robotassisted laminectomy (RAL) was performed on the left side of the lamina $(n=30)$ and $M L$ was performed on the right side $(n=30)$. The time required for laminectomy on one side, whether the lamina was penetrated, and the remaining thickness of the lamina were compared between the two groups.
\end{abstract}

RESULTS The time required for laminectomy on one side was longer in the RAL group than in the ML group (median 326 seconds [IQR 133 seconds] vs 108.5 seconds [IQR 43 seconds], $p<0.001)$. In the RAL group, complete lamina penetration occurred twice (6.7\%), while in the ML group, it occurred 9 times (30\%); the difference was statistically significant $(p=0.045)$. There was no statistically significant difference in the remaining lamina thickness between the two groups (median $1.035 \mathrm{~mm}$ [IQR $0.419 \mathrm{~mm}$ ] vs $1.084 \mathrm{~mm}$ [IQR $0.383 \mathrm{~mm}], \mathrm{p}=0.842$ ).

CONCLUSIONS The results of this study confirm the safety of this novel spinal robot system for laminectomy. However, its efficiency requires further improvement. https://thejns.org/doi/abs/10.3171/2021.10.FOCUS21499

KEYWORDS robot-assisted laminectomy; robotic surgical procedures; state recognition; spine surgery; piezoelectric osteotome system

$\mathrm{P}$ OSTERIOR spine surgery is a common surgical approach for treating spinal diseases such as spinal canal stenosis in the thoracic or lumbar spine. The key procedures of posterior spine surgery mainly include pedicle screw placement for internal fixation, decompressive laminectomy, and fusion. Each step must be performed carefully to avoid damaging the adjacent nerves or vessels, which can sometimes be catastrophic. Over the past 2 decades, spinal robots designed to improve the accuracy of pedicle screw placement have been developing rapidly, and several robots have been successfully used in the clinic. ${ }^{1-3}$ In 2018, Fan et al. concluded in their meta-analysis that robot-assisted techniques have a statistically significant superiority in the accuracy of pedicle screw placement over conventional methods. ${ }^{4}$ In some reports, the accuracy of robot-assisted screw placement was as high as $99 \% .{ }^{5}$ The application of robots in the field of pedicle screw placement has achieved great success. However, decompressive laminectomy, a step that is just as critical as pedicle screw placement, does not have a mature robot-assisted system. ${ }^{6}$

Laminectomy involves several risks, such as nerve root injury, dural injury, cauda equina syndrome, and spinal cord injury. ${ }^{7,8}$ This is partly because the lamina is anatomically located adjacent to these critical structures and partly because of the limited information available to the surgeon when performing a manual laminectomy (ML). Even the most experienced surgeon can only determine whether the lamina has been penetrated by indirect information, such as the feeling of force from the bone-cutting tools. This requires the surgeon not only to be sensitive to this change in

ABBREVIATIONS ML = manual laminectomy; RAL = robot-assisted laminectomy. SUBMITTED August 31, 2021. ACCEPTED October 22, 2021. INCLUDE WHEN CITING DOI: 10.3171/2021.10.FOCUS21499.

* Z.L. and S.J. contributed equally to this work. 


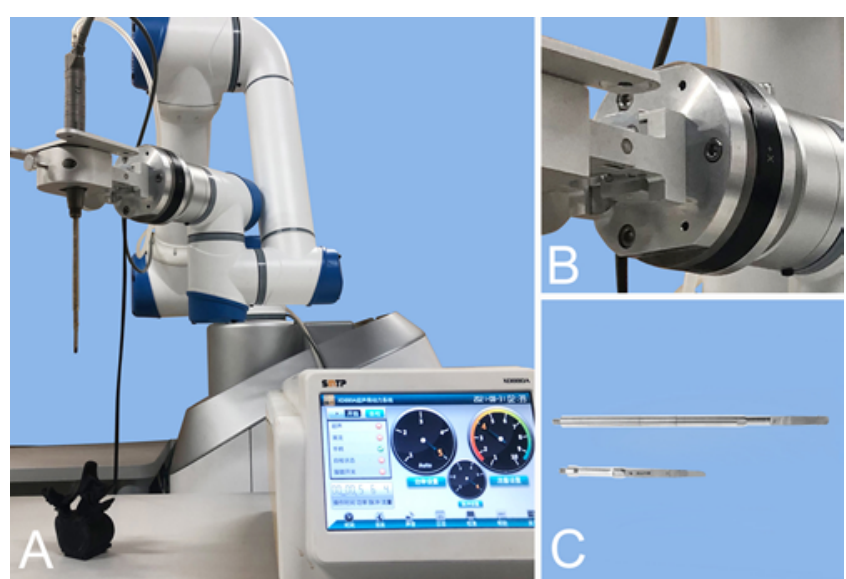

FIG. 1. A: Photograph of the robot system, including a 6 degrees of freedom robotic arm, a force sensor, and a piezoelectric osteotome system. B: Force sensor. C: Special blade for the robot (upper) compared with the hand-held blade (lower); the robot blade is nearly square and is longer.

force but also to be able to stop cutting immediately when such changes are perceived, which can be difficult when the surgeon is tired due to the long operation time. The robot system offers an advantage considering this aspect, as it is more sensitive to changes in force and does not get tired. We designed a collaborative spine robot system to assist with laminectomy. This robot system uses the piezoelectric osteotome system as a bone-cutting tool and monitors whether the lamina will be penetrated based on force information. This preliminary study aimed to explore the reliability of this robot system and compare it with that of ML also using a piezoelectric osteotome system.

\section{Methods \\ Robot Architecture}

This novel robot system comprises an operating system and a control system. The operating system includes a 6 degrees of freedom robotic arm (I5, AUBO Robotic Technology Co., Ltd.) and a force sensor (SRI, Shanghai Yuli Industrial Co., Ltd.) loaded at the end of the robotic arm, combined with a piezoelectric osteotome system (XD880A, SMTP Technology Co., Ltd.) (Fig. 1). The handle of the piezoelectric osteotome system is loaded with a special blade for the robot system, which is $4.5 \mathrm{~mm}$ wide and $0.7 \mathrm{~mm}$ thick. In contrast to a commonly used handheld blade, its shape is deformed from oblique to nearly square in order to provide more valuable force information in the vertical direction. It is also $7 \mathrm{~cm}$ longer than the handheld blade, to prevent the robotic arm from contacting the vertebrae and affecting the cutting process (Fig. 1C). The control terminal of the piezoelectric osteotome system is connected to a workstation, forming the control system of the robot. The workstation runs a specially designed software to achieve intraoperative interaction with the operating system to control the operation, including aspects such as the movement of the robotic arm or adjustment of the parameters of the piezoelectric osteotome system. The robot system is designed as a collaborative system. The operator needs to position the robot manu- ally before cutting, and issue instructions at the beginning and end of the laminectomy. The cutting procedure is performed automatically by the robot while under supervision.

\section{Cutting Strategy}

The robot is designed to cut vertically step-by-step, and its safety strategy is based on force information. Specifically, the operator first suspends the blade above the cephalic side of the cutting path of the laminectomy manually. Subsequently, the robot performs vertical cutting at a speed of $0.5 \mathrm{~mm}$ per second until the system recognizes that the lamina will be penetrated based on the information received by the force sensor. Next, the robotic arm is lifted and moved $4 \mathrm{~mm}$ ( $<$ the width of 1 blade) along the cutting path toward the caudal side. If no stop command is received, the robot enters the next vertical cutting step after a short pause. Video 1 shows 2 vertical cuttings, including the 4 processes of 1 step of vertical cutting: cutting, stop cutting, lifting, and moving forward.

VIDEO 1. Video of 2 steps of vertical cutting. Each cutting step consists of starting the cutting (moving vertically down), stopping the cutting (the robot system recognizes that it is about to penetrate the lamina), lifting up, and moving forward $4 \mathrm{~mm}$. If no stop command is received after these 4 steps, after a short pause, the robot enters the next cutting step and continues to cycle until the operator considers that the cut has been completed and sends the stop command. The upper-right corner of the video shows the robot's operating software. This specially designed software presents force information in real time to facilitate operator monitoring. Copyright Weishi Li. Published with permission. Click here to view.

\section{Operation Process}

The overall operation process for a one-sided laminectomy performed using a robotic system is as follows. 1) The lumbar specimen is fastened to the fixture (Fig. 2A). The surgeon moves the mechanical arm manually so that the blade is hovering over the cutting site, usually the cephalic side of the lamina, and the blade orientation is consistent with the cutting track (Fig. 2B). 2) The cutting direction (to the cephalic side or the caudal side) is set on the workstation. The operation is started under the supervision of the surgeon. The robot gradually performs cutting according to the aforementioned vertical cutting strategy (Fig. 2C and Video 1). The operator can stop the operation at any time using the emergency stop button of the robot. 3) When the robot completes the last vertical cutting of the caudal side of the lamina, the assistant stops the robotic operation through the workstation.

\section{Study Design}

The experiment was performed on 30 in vitro porcine lumbar vertebral specimens. After euthanizing the Guangxi Bama minipigs (pigs that had the lumbar vertebrae still intact after other research projects), specimens of the lumbar vertebrae were obtained and dissected into individual vertebrae to fully expose the outside of the lamina. The vertebral body was removed to fully expose the inner side of the lamina (Fig. 2A). The experiment was performed within 1 day of specimen collection. A total of 30 lumbar vertebrae specimens were obtained from 


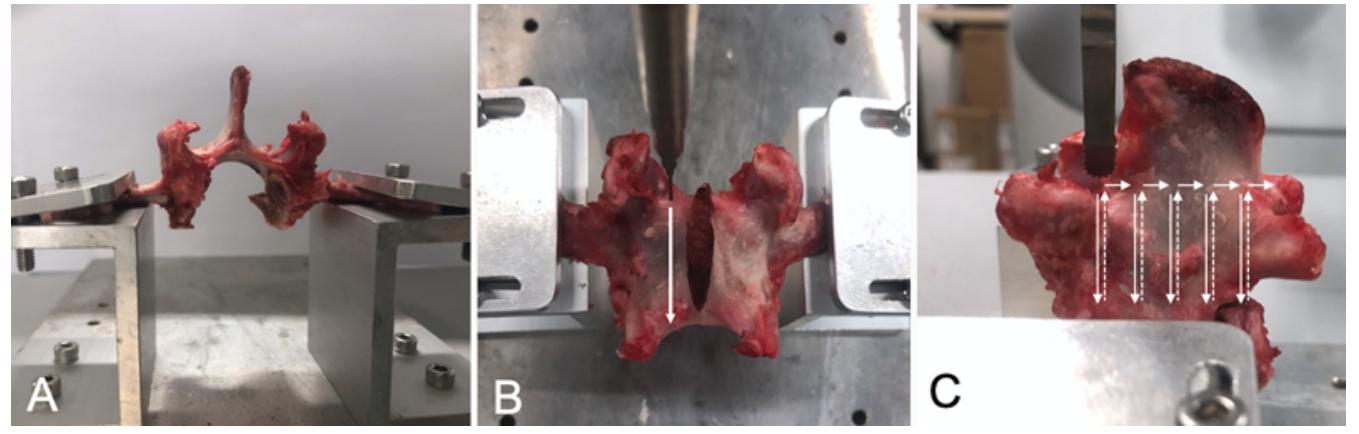

FIG. 2. Photographs of the operation process for this robot system. A: The lumbar specimen, with the vertebral body removed, is fastened to the fixture. B: Movement of the blade to the specified position is performed manually. The blade orientation is kept consistent with the cutting track (white arrow). C: The robot automatically performs the laminectomy following a vertical cutting strategy (white arrows) until it receives the operator's stop command.

L1-6 of 5 pigs aged 12 months (30-35 kg). Robot-assisted laminectomy (RAL) was performed on the left side of the lamina, while ML was performed on the right side using a piezoelectric osteotome system with the same blade as the robot system. Although the blade is optimized for the robot, it can also be used during manual operation. ML was performed by an attending physician with 1 year of experience, who was also required to avoid penetrating the lamina to the maximum possible extent. The experimental protocol was reviewed and approved by the ethics committee of our hospital. To comprehensively evaluate the effect of the laminectomy, we analyzed it from the following 3 perspectives. 1) The time to complete the laminectomy on one side, measured as the time required from the first contact of the blade with the outer cortical bone of the lamina to the completion of the laminectomy. 2) The lamina penetration status, whereby the penetration status of 1 vertical cut could be divided into no penetration, incomplete penetration, and complete penetration. If the length of the penetration hole was $<4.5 \mathrm{~mm}$ (the width of the blade), it was classified as incomplete penetration. In this case, the procedure was still considered safe because even in the case of partial penetration, the invasion of the blade into the spinal canal is minimal. If the length of the penetration hole was $\geq 4.5 \mathrm{~mm}$, it was considered complete penetration, which was unsafe because judging how far the blade had intruded into the spinal canal was not possible. Therefore, if there was no complete penetration during the laminectomy, we defined the lamina penetration status for that laminectomy as grade A, meaning that the procedure was safe. On the contrary, if complete penetration occurred, it was defined as grade $\mathrm{B}$, meaning unsafe (Fig. 3). 3) The remaining lamina thickness, whereby the cutting plane of the lamina was divided into 4 equal parts and the thicknesses of the 5 boundaries were measured. Finally, the average value was considered as the thickness of the remaining lamina.

\section{Statistical Analysis}

Continuous variables not subject to normal distribution are presented as the median and IQR and were compared between the groups using the Mann-Whitney U-test. Categorical variables are presented as percentages and were analyzed using the chi-square test. IBM SPSS Statistics version 24.0 (IBM Corp.) was used for the data analysis. Statistical significance was set at $\mathrm{p}<0.05$.

\section{Results}

The median time taken for a single-sided laminectomy
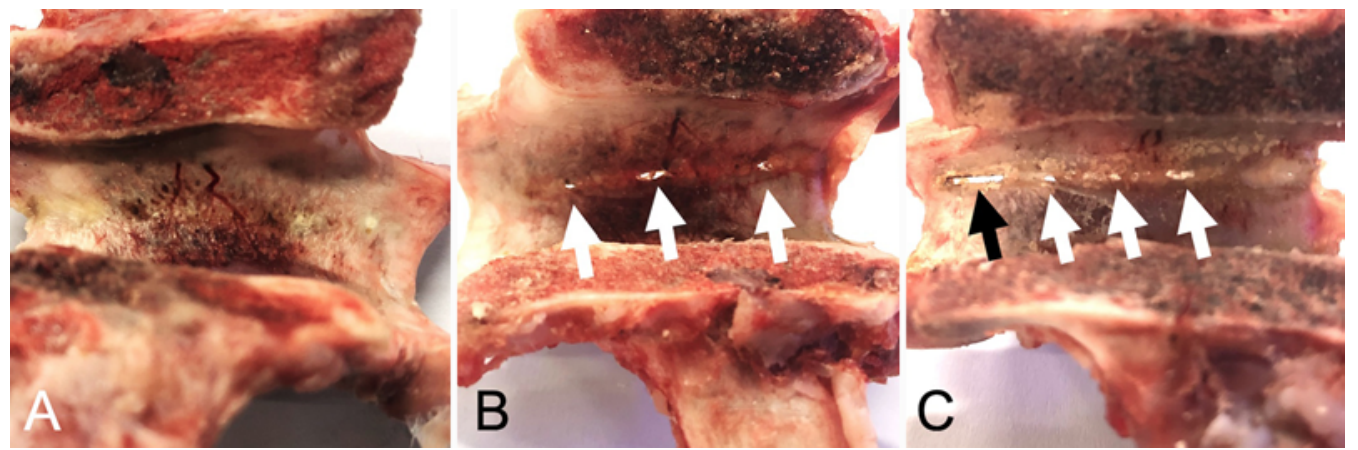

FIG. 3. Photographs of the lamina penetration condition. A: No penetration holes are observed from the inner side of the lamina, which is defined as grade A, signifying safety. B: Several incomplete penetration holes can be observed (white arrows), as the length of these penetration holes are $<4.5 \mathrm{~mm}$ (the width of the blade). This condition is also defined as grade A, signifying safety. C: Both complete penetration (black arrow; the penetration hole $=4.5 \mathrm{~mm}$ ) and incomplete penetration (white arrows) can be observed, which is defined as grade B and unsafe. 
TABLE 1. Comparison between RAL and ML groups

\begin{tabular}{|c|c|c|c|}
\hline Variable & $\begin{array}{l}\text { RAL Group } \\
(n=30)\end{array}$ & $\begin{array}{l}\text { ML Group } \\
(n=30)\end{array}$ & $p$ Value \\
\hline $\begin{array}{l}\text { Median time for laminec- } \\
\text { tomy, sec }(I Q R) \dagger\end{array}$ & $326.0(133.0)$ & $108.5(43.0)$ & $<0.001^{*}$ \\
\hline $\begin{array}{l}\text { Median remaining thickness } \\
\text { of the lamina, } \mathrm{mm}(\mathrm{IQR})\end{array}$ & $1.035(0.419)$ & $1.084(0.383)$ & 0.842 \\
\hline Penetration, n (\%)‡ & & & $0.045^{\star}$ \\
\hline Grade A & $2(6.7)$ & $9(30)$ & \\
\hline Grade B & $28(93.3)$ & $21(70)$ & \\
\hline \multicolumn{4}{|c|}{$\begin{array}{l}{ }^{*} p<0.05 \text {, statistically significant. } \\
\dagger \text { Time taken for one-sided laminectomy. } \\
\ddagger \text { Grade A indicates that no penetration or only incomplete penetration (the } \\
\text { length of the penetration point is }<4.5 \mathrm{~mm} \text { ) was observed from the inner side } \\
\text { of lamina, signifying that the laminectomy was safe. Grade B indicates that } \\
\text { complete penetration (the length of the penetration point is } \geq 4.5 \mathrm{~mm} \text { ) was } \\
\text { observed, signifying that the laminectomy was unsafe. }\end{array}$} \\
\hline
\end{tabular}

was 326 seconds (IQR 133 seconds) in the RAL group versus 108.5 seconds (IQR 43 seconds) in the ML group $(\mathrm{p}<0.001)$. Furthermore, there was no significant difference in the median residual lamina thickness between the two groups $(1.035 \mathrm{~mm}$ vs $1.084 \mathrm{~mm}, \mathrm{p}=0.842)$. Complete lamina penetration (grade B) occurred twice $(6.7 \%)$ in the RAL group and nine times (30\%) in the ML group; the difference was statistically significant $(p=0.045)$ (Table 1$)$.

\section{Discussion}

No mature robot system has yet been reported in the field of RAL. A safer way to perform laminectomy is not to cut through the lamina directly but to stop the laminectomy when the depth is "about to penetrate" the lamina. The breaking of the lamina is left to the surgeon, which can help avoid nerve root or epidural injury caused by deep cutting. The longitudinal lamina structure can be simplified as a sandwich model of outer cortical bonemiddle cancellous bone-inner cortical bone. If the robot can recognize these 3 layers and stop just before penetrating the inner cortical bone layer, the safety of the RAL can be guaranteed. Most researchers have focused on the strategy of state recognition in robot systems. In 2010, Wang et al. ${ }^{9}$ used force information during laminectomies as the basis for state recognition and identified different bone layers by calculating the cross-correlation coefficient to stop milling when the inner cortical bone of the lamina was reached. In 2015, Dai et al. ${ }^{10}$ identified different milling states by using a series of bandpass filters based on the wavelet packet transform to analyze the sound pressure signal, which was collected during a laminectomy through a microphone. In addition, they also attempted to use the vibration harmonic amplitude during the milling process to identify different milling states. ${ }^{11}$ However, during an actual operation, the surrounding environment may interfere with the sound. Furthermore, due to the limited surgical space, it is challenging to collect vibration information during laminectomy. In contrast, information on the force is stable and easy to obtain. Therefore, in this study, we selected force information as the basis for state recognition. The fuzzy control mechanics model was established by normalizing the vertical force information, and state identification was finally realized.

In their study, Wang et al. ${ }^{9}$ showed that the time for a single-sided laminectomy was 10 to 14 minutes. In our study, the time for a single-sided RAL was nearly 5.5 minutes, which was significantly shorter. Although there remains a gap compared with the ML group, which is due to the current robot's vertical cutting speed being set to 0.5 $\mathrm{mm}$ per second, this time gap can be improved further. As for the bone-cutting strategy, most previous studies have chosen layer-by-layer grinding, which is limited by the grinding drill ${ }^{9,11-13}$ and is overall inefficient. To the best of our knowledge, this is the first study to use the piezoelectric osteotome system as a bone-cutting tool in RAL for the purpose of adopting a vertical cutting method.

In this study, laminectomy was performed using the robotic system, which successfully identified the inner cortical bone 28 times (93.3\%) without completely penetrating the lamina, compared with 21 times $(70 \%)$ in the ML group. This is because, in ML, only the moment of penetration can be judged when the lamina has already been penetrated. However, in RAL, the robot can identify not only the moment of penetration but also the moment it contacts the inner cortex bone layer, allowing the robot to react a step faster than surgeons. From this perspective, RAL is safer than ML.

The cutting depth of the lamina, as a parameter, was also important in this study. If the cutting depth is insufficient, the force required to break off the lamina will eventually increase, increasing the patient's risk. Therefore, we compared the thickness of the last remaining lamina between the two groups and found no significant differences. Approximately $1 \mathrm{~mm}$ was left in both groups. This means that the laminectomy performed by the robot was adequate in depth, which verifies the safety of the robot in this aspect.

The robot automation level grading system proposed by Yang et al. ${ }^{14}$ (levels 0-5) includes level 0, no autonomy; level 1, robot assistance; level 2, task autonomy; level 3, conditional autonomy; level 4, high autonomy; and level 5, full autonomy (no human needed). According to this grading system, robot automation in the field of RAL is currently at levels 0 to $2 .{ }^{6}$ Ponnusamy et al. ${ }^{15}$ reported an attempt to perform laminectomy using the Da Vinci robot to perform posterior spinal surgery in animal experiments. The Da Vinci robot is a level 0 robot, a type of remote robot with movements that are entirely dependent on the operator, with no degree of automation. A level 1 robot provides only mechanical guidance or assistance, whereas a human has continuous control of the system. For example, the robot reported by Hein et al. ${ }^{16}$ and Francoise et al. ${ }^{17}$ adopted a level 1 virtual fixture technology. According to the danger area plan, the surgeon and the robot hold the grinding drill together during the operation and the robot is only used to ensure that the end of the bone-cutting tool does not enter the danger area. Level 2 autonomous robots can perform some operations autonomously under human supervision. This is common in recent research related to RAL. Although there is no mature robotic system, the operating platform used in these studies has achieved 
this level of automation, as it can automatically perform laminectomy. ${ }^{9,11,12,18-20}$ The robot system we developed was at a level of 2. Choosing this level of automation is reasonable because robots have better perception than humans. Our results also verified the safety of a laminectomy that was automatically performed by the robot system to some extent.

This study has some limitations. First, the robot's positioning needs to be carried out manually, and the robot cannot autonomously identify the position of the lamina, a process that takes time. Second, this study was only carried out in vitro, and, thus, its application scenarios are quite different from real-world clinical applications. For example, in real-life situations, the lamina will move with the patient's breathing movements, which will lead to more complicated changes in the vertical force information and interfere with the robot's judgment. Therefore, subsequent research needs to combine medical image registration and navigation to achieve the goal of automatic lamina recognition by the robot. Simultaneously, the compensation for respiratory movement should be improved to be closer to the actual clinical operating environment.

\section{Conclusions}

In this study, we report a novel collaborative robot system for laminectomy with a safety strategy based on force information. Although the cutting efficiency requires further improvement, the cutting safety of this robotic system is acceptable. These results confirm the feasibility of RAL and provide a foundation for subsequent studies.

\section{Acknowledgments}

This work was funded by the National Key Research and Development Program of China (2018YFB1307604) and the Natural Science Foundation of Beijing, China (no. L202010).

\section{References}

1. Jiang B, Azad TD, Cottrill E, et al. New spinal robotic technologies. Front Med. 2019;13(6):723-729.

2. Elswick CM, Strong MJ, Joseph JR, Saadeh Y, Oppenlander M, Park P. Robotic-assisted spinal surgery: current generation instrumentation and new applications. Neurosurg Clin N Am. 2020;31(1):103-110.

3. Huang J, Li Y, Huang L. Spine surgical robotics: review of the current application and disadvantages for future perspectives. J Robot Surg. 2020;14(1):11-16.

4. Fan Y, Du JP, Liu JJ, et al. Accuracy of pedicle screw placement comparing robot-assisted technology and the free-hand with fluoroscopy-guided method in spine surgery: an updated meta-analysis. Medicine (Baltimore). 2018;97(22):e10970.

5. Huntsman KT, Ahrendtsen LA, Riggleman JR, Ledonio CG. Robotic-assisted navigated minimally invasive pedicle screw placement in the first 100 cases at a single institution. $J$ Robot Surg. 2020;14(1):199-203.

6. Li Z, Yu G, Jiang S, Hu L, Li W. Robot-assisted laminectomy in spinal surgery: a systematic review. Ann Transl Med. 2021; 9(8):715.

7. Carroll SE, Wiesel SW. Neurologic complications and lumbar laminectomy. A standardized approach to the multiplyoperated lumbar spine. Clin Orthop Relat Res. 1992;(284): 14-23.
8. Mayfield FH. Complications of laminectomy. Clin Neurosurg. 1976;23:435-439.

9. Wang T, Luan S, Hu L, Liu Z, Li W, Jiang L. Force-based control of a compact spinal milling robot. Int J Med Robot. 2010;6(2):178-185.

10. Dai Y, Xue Y, Zhang J. Bone milling condition monitoring based on sound signal processing. J Vibration Shock. 2015 2015;34(22):19-23.

11. Dai Y, Xue Y, Zhang J. Vibration-based milling condition monitoring in robot-assisted spine surgery. IEEE ASME Trans Mechatron. 2015;20(6):3028-3039.

12. Deng Z, Zhang H, Guo B, et al. Hilbert-Huang transform based state recognition of bone milling with force sensing. In: 2013 IEEE International Conference on Information and Automation. IEEE; 2013:937-942.

13. Wang $\mathrm{S}, \mathrm{Gao} \mathrm{P}, \mathrm{Fan} \mathrm{L}$, et al. A bone milling robot for spinal surgery. J Med Devices. 2016;10(3):030924.

14. Yang GZ, Cambias J, Cleary K, et al. Medical robotics-regulatory, ethical, and legal considerations for increasing levels of autonomy. Sci Robot. 2017;2(4):eaam8638.

15. Ponnusamy K, Chewning S, Mohr C. Robotic approaches to the posterior spine. Spine (Phila Pa 1976). 2009;34(19):21042109.

16. Hein A, Lueth TC. Control algorithms for interactive shaping surgical robots. In: Proceedings 2001 ICRA IEEE International Conference on Robotics and Automation. IEEE; 2001: 2025-2030.

17. Francoise V, Sahbani A, Morel G. A comanipulation device for orthopedic surgery that generates geometrical constraints with real-time registration on moving bones. In: 2011 IEEE International Conference on Robotics and Biomimetics. IEEE; 2011:38-43.

18. Dai Y, Xue Y, Zhang J. Milling state identification based on vibration sense of a robotic surgical system. IEEE Trans Ind Electron. 2016;63(10):6184-6193.

19. Fan L, Gao P, Zhao B, et al. Safety control strategy for vertebral lamina milling task. CAAI Trans Intell Technol. 2016; 1(3):249-258.

20. Sun Y, Jiang Z, Qi X, et al. Robot-assisted decompressive laminectomy planning based on 3D medical image. IEEE Access. 2018;2018(6):22557-22569.

\section{Disclosures}

The authors report no conflict of interest concerning the materials or methods used in this study or the findings specified in this paper.

\section{Author Contributions}

Conception and design: W Li, Z Li, Hu. Acquisition of data: Z Li, Jiang, Song, Liu, Wang. Analysis and interpretation of data: Z Li, Jiang, Liu, Wang. Drafting the article: Z Li. Critically revising the article: $\mathrm{Z} \mathrm{Li}$, Jiang, Hu. Reviewed submitted version of manuscript: W Li, Z Li, Jiang. Approved the final version of the manuscript on behalf of all authors: W Li. Statistical analysis: Z Li, Jiang, Liu. Administrative/technical/material support: W Li, Song, Hu. Study supervision: W Li, Z Li.

\section{Supplemental Information \\ Videos}

Video 1. https://vimeo.com/643441631.

\section{Correspondence}

Weishi Li: Peking University Third Hospital, Beijing, China. puh3liweishi@163.com. 